\title{
RITMOS DEL SER Y LA ANTINOMIA DEL AFECTO: SOBRE SPINOZA Y DELEUZE $^{1}$
}

\author{
Rhythms of Being and the Antinomy of Affect: on Spinoza and Deleuze \\ Donald Cross \\ Pontificia Universidad Católica de Chile, Santiago, Chile \\ docross@uc.cl
}

\section{Resumen}

Este artículo tiene tres partes. La primera desarrolla la individuación corpórea como función del movimiento y reposo en Spinoza. Basándome en los comentarios de Deleuze, describo el conjunto de estos movimientos corpóreos como una "coreografía existencial". Sin embargo, Deleuze y Spinoza no están completamente de acuerdo sobre la naturaleza del cuerpo o su afectabilidad. En la segunda parte, abordo este desacuerdo como una antinomia: según la ley "espinosista", un afecto no puede afectar la esencia del afectado; según la ley "deleuziana", el afecto tiene que hacer que el afectado devenga otro. En la tercera parte, me detengo en esta noción deleuziana del "devenir" afectivo. En la medida en que el devenir conlleva una desincorporación total del afecto, planteo que, para romper con el tratamiento tradicional del afecto, Deleuze tiene que repetirlo de manera aún más brutal.

Palabras clave: Deleuze, afecto, cuerpo, arte, materialismo.

\begin{abstract}
This article has three parts. The first elaborates corporal individuation as a function of movement and rest in Spinoza. Drawing upon Deleuze's commentaries, I describe the entirety of these corporal movements as an "existential choreography." Yet, Deleuze and Spinoza don't entirely agree about the nature of the body or its affectability. In the second part, I address this disagreement as an antinomy: according to the "Spinozist" law, an affect cannot affect the essence of what it affects; according to the "Deleuzian" law, affect must make the affected become other. In the third part, I look more closely at this Deleuzian notion of affective "becoming." Insofar as becoming entails a total disembodiment of affect, I argue that, in order to break with the traditional treatment of affect, Deleuze must replicate it more brutally still.
\end{abstract}

Keywords: Deleuze, affect, body, art, materialism.

Fecha de Recepción: 01/07/2019 - Fecha de Aceptación: 26/11/2019

\footnotetext{
${ }^{1}$ Versión ampliada y revisada de la ponencia dictada el 13 de diciembre del 2018 en el coloquio Cuerpos espacios movimientos: entre arquitectura, filosofía y danza, organizado por Andrea Potestà y el Grupo de Investigación Cuerpo y lenguaje del Instituto de Filosofía de la Pontificia Universidad Católica de Chile. Le agradezco a Andrea por su invitación a participar en el coloquio. Además, le agradezco a Paula Cucurella por su corrección y lectura paciente de este texto.
} 


\section{Los ritmos}

Si lo que nos orienta aquí es el "cuerpo" y el "movimiento", existe una razón específica para darle un lugar privilegiado a Spinoza en nuestra discusión. Para Spinoza, el movimiento define al cuerpo.

Spinoza plantea que hay una sola substancia, la "Naturaleza" o "Dios". La substancia consta de una infinitud de atributos, pero nosotros, como seres finitos, seres humanos, sólo conocemos dos: el pensamiento y la corporeidad. Con más tiempo, tendría que detenerme indefinidamente en la determinación espinosista de la corporeidad o la extensión como atributo divino ${ }^{2}$, pero una sola consecuencia me interesa destacar de este problema. Si la corporeidad es un atributo de Dios, de la única substancia, entonces no hay ninguna diferencia substancial entre los cuerpos individuales, los cuerpos determinados, a los que Spinoza denomina "modos". Mi cuerpo, el vuestro, todo cuerpo es una modificación de la misma corporeidad de la misma substancia. Todos los cuerpos son igualmente corpóreos. Pero, entonces, ¿cómo se distinguen los cuerpos entre sí?

Cito el primer Lema de la segunda parte de la Ética: "Los cuerpos se distinguen entre sí en razón del movimiento y el reposo, de la rapidez y la lentitud, y no en razón de la substancia". Un cuerpo determinado, según Spinoza, consta de cuerpos más sencillos. Estos cuerpos más sencillos o se mueven o reposan. Un cuerpo se define y dura, como el cuerpo determinado que es, mientras los cuerpos que lo componen mantengan una relación fija entre movimiento y reposo. Los cuerpos componentes pueden intercambiarse (Lema 4), unos pueden acelerar y otros lentificarse (Lema 5), sus tamaños pueden variar (Lema 6), pero el cuerpo entero sigue siendo el mismo cuerpo si la relación general entre movimiento y reposo sigue igual. Un cuerpo no se distingue de otro ni substancialmente ni ontológicamente; un cuerpo no es otro, mi cuerpo no es el tuyo, mi cuerpo no es un árbol ni una estrella porque los cuerpos que componen mi cuerpo mantienen una relación particular y constante entre movimiento y reposo, entre rapidez y lentitud.

En último término, si sopesamos esta relación constitutiva entre el cuerpo y el movimiento, ya no podemos hablar de "cuerpos en movimiento" ${ }^{3}$, si esta formulación presupone "cuerpos" ya constituidos, y que luego entrarían en movimiento. Ya no se trata de cuerpos en movimiento, sino de movimientos en el

\footnotetext{
${ }^{2} C f$., en particular, Spinoza, Ética, Parte I, Proposición 15, Escolio.

${ }^{3}$ Subtítulo del segundo día del coloquio en que se presentó la ponencia base de este artículo.
} 
cuerpo. O, mejor dicho, de movimientos como cuerpo. Spinoza nos obliga, pues, a distinguir entre el movimiento y el movimiento: por un lado, está el movimiento que un cuerpo emprende; por otro, el movimiento que constituye al cuerpo como el cuerpo que es. Un cuerpo no puede moverse sin ya haber sido constituido por otro movimiento, un movimiento anterior, un movimiento antes de su movimiento. Por lo tanto, habría que hablar de un movimiento pre- o proto-móvil o incluso de un movimiento inmóvil, ya que los movimientos que constituyen el cuerpo no lo mueven en el sentido común. Sea lo que sea que soy, antes de moverme, sin moverme, soy movimiento.

Sobre la base de la distinción entre movimiento constitutivo y movimiento constituido, entre el movimiento que constituye al cuerpo y el movimiento que el cuerpo constituido emprende, podría seguir fabricando formulaciones paradójicas como "movimiento pre-móvil" y "movimiento inmóvil". Pero en algún momento habría que recordar que, para Spinoza, la constitución del cuerpo no consiste en puro movimiento sino más bien en una relación entre movimiento y reposo. Así, uno tendría que ampliar el término "movimiento" para referirse al movimiento y al reposo, o bien buscar otro término que abarcara mejor ambas cosas.

Un término posible - aunque no es el único- podría ser la danza.

Para desarrollar una noción de danza en este sentido amplio, un espinosista resulta tan imprescindible como el propio Spinoza. Desde 1978 hasta 1993, desde Spinoza: filosofía práctica hasta "Spinoza y las tres 'Éticas'", el último texto del último libro que publicó durante su vida (Crítica y clínica), Deleuze utiliza la palabra ritmo para referirse a la relación entre movimiento y reposo constitutiva del cuerpo espinosista ${ }^{4}$ La palabra es afortunada, pues, ¿qué es la relación entre reposo y movimiento, velocidad y lentitud, sino un ritmo? Y ¿qué es un ritmo corporal sino una danza?

Casi inmediatamente después de introducir la palabra "ritmo" en "Spinoza y las tres 'Éticas'", es verdad que el propio Deleuze se refiere a la danza como un ejemplo de composición corpórea entre otros. Deleuze entiende el ritmo en un sentido más amplio, más fundamental, más universal ${ }^{5}$, pero sigue pensando la danza como una relación entre movimiento y reposo en particular. Como una actividad determinada que ocupa un nivel en la escala que comienza con los cuerpos más simples y sigue con los cuerpos más complejos, hasta el cuerpo

\footnotetext{
${ }^{4}$ Deleuze 1981 163/2001 151, 1993 176/1996 224 (la primera cita se refiere al texto francés, la segunda a la traducción española).

5 "La estructura o el objeto está formado por dos cuerpos cuanto menos, cada uno de ellos formado por dos o más cuerpos hasta el infinito, que se unen en el otro sentido en cuerpos cada vez más amplios y compuestos, hasta el objeto único de la Naturaleza entera, estructura infinitamente transformable y deformable, ritmo universal, Facies totius Naturae, modo infinito" (Deleuze 1993 177/1996 224).
} 
compuesto de todos los cuerpos, a saber, la Naturaleza ${ }^{6}$. Dicho de otro modo, para Deleuze, la danza es un ejemplo de la manera en que los ritmos de mi cuerpo pueden ser combinados con los de otro cuerpo para formar un ritmo corpóreo más complejo. "Si aprendo a nadar, o a bailar, - dice Deleuze- es preciso que mis movimientos y mis pausas, mis velocidades y mis lentitudes adquieran un ritmo común con los del mar, o de la pareja, siguiendo un ajuste más o menos duradero" (1993 176/1996 224). Sin duda valdría la pena aceptar la invitación de Deleuze a pensar la danza de dos parejas en términos espinosistas, pero me parece que podría haber una danza más fundamental que la que se efectúa entre dos cuerpos humanos. Si todo cuerpo se define por un ritmo de movimiento y reposo, y si todo ritmo corpóreo es danza, el resultado es una generalización onto-existencial de la danza.

Tal danza general ya no se limita a la danza que hago con mayor o menor elegancia cuando comienza la música. Ya no se limita a la danza que hago con una pareja. Esta danza ya no se limita. En la medida en que todo cuerpo se define por ritmos de movimiento y reposo, desde los cuerpos más sencillos hasta la Naturaleza en su conjunto, la danza ya no se contiene dentro de ninguna región determinada de la Naturaleza. Más bien, como la condición de toda definición corpórea, la danza describe la Naturaleza misma y circunscribe sus regiones. Todo cuerpo, pues, es fundamental y primariamente danza, de modo que la danza ya no se distingue del cuerpo que danza. Toda raíz, toda hoja, todo árbol; todo mineral, todo sedimento, toda piedra; todo dedo, toda mano, todo brazo; literalmente todo el mundo danza al ser lo que es y hacer todo lo que hace. En este punto nos topamos con lo que podría denominarse -sin metáfora alguna- una coreografía existencial: antes de que yo dé un paso, mi cuerpo ya es coreografía. Lo que significa que no muero al convertirme en cadáver ${ }^{7}$. Aún si la sangre sigue circulando, muero cuando -y sólo cuando- se acaba la danza. Bailamos todos - sin excepción alguna - hasta la muerte. Es decir, hasta que comience otra danza que ya no es la mía. Otra danza que no soy yo ${ }^{8}$.

No diré más sobre esta coreografía existencial. No insistiré en la manera en que Deleuze concuerda con Spinoza, la cual suele darse por sentado en la bibliografía secundaria, porque me interesa más destacar un desacuerdo tácito

${ }^{6}$ Cf. Spinoza, Ética, Parte II, Lema 7, Escolio.

7 "En efecto: ninguna razón me impele a afirmar que el cuerpo no muere más que cuando es ya un cadáver" (Spinoza, Ética, Parte IV, Proposición 39, Escolio).

${ }^{8}$ Dicho de otra manera, la danza no se acaba en la medida en que no hay descomposición desde la perspectiva de la naturaleza entera: "Pues, desde el punto de vista de la naturaleza o de Dios, se da en todo caso composición de relaciones, y nada más que composición de relaciones, según las leyes eternas" (Deleuze 1981 51/2001 48). 
entre ambos filósofos acerca del cuerpo y su afectabilidad, esto es, la capacidad del cuerpo de afectar y de ser afectado. Digo "tácito", un desacuerdo tácito, porque el propio Deleuze no dice nada al respecto. No dice nada a pesar de que, y tal vez precisamente porque, escribió de manera voluminosa sobre Spinoza, y plantea que Spinoza encarna la existencia y la posibilidad misma de la filosofía. "Cristo de los filósofos", le dice (1991 62/1993 62). Tácito o no, este desacuerdo tiene consecuencias para toda la historia de la filosofía y, en particular, para los lugares irreductibles de Spinoza y de Deleuze en esa historia.

\section{La antinomia}

A lo largo de la Ética, y con la fuerza de todo su sistema, Spinoza no se cansa de denunciar el antropocentrismo. Ya en el apéndice de la primera parte, por ejemplo, no sólo niega que la naturaleza gire en torno al ser humano, sino que también demuestra la razón por la cual la idea es inevitable.

Todos los prejuicios que intento indicar aquí dependen de uno solo, a saber: el hecho de que los hombres supongan, comúnmente, que todas las cosas de la naturaleza actúan, al igual que ellos mismos, por razón de un fin, e incluso tienen por cierto que Dios mismo dirige todas las cosas hacia un cierto fin, pues dicen que Dios ha hecho todas las cosas con vistas al hombre, y ha creado al hombre para que le rinda culto (Spinoza, Ética, Parte I, Apéndice, 109)9.

Sin embargo, aunque sus criticas del antropocentrismo son precoces y duraderas, cabe destacar que Spinoza no abandona todo privilegio del ser humano. Al contrario, la crítica espinosista del hombre se efectúa con el fin de precisar otro privilegio. Así, se podría describir el lugar del ser humano en el sistema espinosista en términos de los dos sentidos del "fin del hombre" que Jacques Derrida desarrolla para describir el pensamiento occidental en general: "fin" como término (la muerte del hombre en sentido tradicional) y "fin" como meta (el hombre en el sentido nuevo que habría que alcanzar ${ }^{10}$.

En efecto, toda la discusión del cuerpo como movimiento en la segunda parte de la Ética, en la cual se basan mis comentarios sobre la coreografía existencial, se emprende "para determinar qué es lo que separa al alma [o Mente: Mens] humana de las demás y en qué las aventaja" (Spinoza, Ética, Parte II, Proposición 13, Escolio). En vez de perderme en los detalles de los axiomas, lemas

9 Asimismo, Spinoza señala el origen fisiológico de todo antropomorfismo con una formulación debidamente famosa: "formará cada cual, según la disposición de su cuerpo, imágenes universales acerca de las demás cosas" (Parte II, Proposición 40, Escolio).

${ }^{10}$ Cf. Derrida 1972 129-64/1994 145-74. 
y postulados que siguen, me limito a una breve descripción del principio afectivo que anima la discusión y funda el privilegio de la mente humana. Según el propio Spinoza:

Con todo, diré en general que, cuanto más apto es un cuerpo que los demás para obrar o padecer muchas cosas a la vez, tanto más apta es su alma [Mente: Mens] que las demás para percibir muchas cosas a la vez; y que cuanto más dependen las acciones de un cuerpo de ese solo cuerpo, y cuanto menos cooperan otros cuerpos con él en la acción, tanto más apta es su alma para entender distintamente (Ibid.).

La perspicacia de la mente, pues, es una función de la afectabilidad del cuerpo. Cuanto más un cuerpo puede obrar y padecer, tanto más la mente puede percibir y eventualmente entender. La capacidad de obrar y padecer del cuerpo, a su vez, depende de su composición, es decir, de los movimientos y reposos que lo componen. De la "danza" en el sentido amplio que elaboré anteriormente. Un cuerpo complejo sería capaz de padecer varios cambios en sus movimientos constitutivos sin perder la relación general, es decir, sería capaz de padecer mucho sin que la interacción lo descomponga. De ahí la excelencia humana. La mente humana aventaja a las demás porque el cuerpo humano es más afectable; se compone de muchísimos cuerpos, muchísimos movimientos, que le dan una especie de latitud de experiencia ${ }^{11}$.

Ahora bien, Spinoza no es ni el primer ni el último filósofo en privilegiar al ser humano. Uno podría decir que no existe filosofía alguna, ni de hecho ni por derecho, sin este problemático privilegio. La única innovación de la Ética al respecto son los términos. Si me he detenido en la forma y plataforma del privilegio en la Ética, es porque ilumina de manera económica una discrepancia importante entre Spinoza y Deleuze en cuanto al afecto: mientras el afecto se introduce en la Ética con el fin de fundar el privilegio del ser humano, el afecto deleuziano pareciera impedir todo privilegio humano. Y lo impediría por definición. Más de una vez en ¿Qué es la filosofía?, el afecto se define como "el devenir no

\footnotetext{
${ }^{11}$ De los postulados al final de la excursión de la Parte II en la corporeidad, los cuales consolidan el argumento, cito los tres más relevantes:

"I. El cuerpo humano se compone de muchísimos individuos (de diversa naturaleza), cada uno de los cuales es muy compuesto".

"III. Los individuos que componen el cuerpo humano (y, por consiguiente, el cuerpo humano mismo) son afectados de muchísimas maneras por los cuerpos exteriores".

"VI. El cuerpo humano puede mover y disponer los cuerpos exteriores de muchísimas maneras".
} 
humano del hombre" (Deleuze y Guattari 1991 169, 173, 184/1993 170, 174, 185) ${ }^{12}$. La fundación de la excelencia del ser humano para Spinoza -el afecto-es el devenir no humano del ser humano para Deleuze. Ahora, la palabra "devenir" tiene un sentido muy técnico para Deleuze, pero antes de intentar precisarlo, quiero abordar de modo más general ambas perspectivas, la espinosista y la deleuziana, para extrapolar lo que llamaré la antinomia del afecto.

Como el nombre indica, una "antinomia" consiste en un conflicto (anti-) entre dos leyes o principios (-nomos). En este caso un conflicto entre lo que llamaré provisionalmente la ley "espinosista" y la ley "deleuziana". Ni Spinoza ni Deleuze tematizan esta antinomia, y no se limita ni a Spinoza ni a Deleuze, pero su encuentro depara una ocasión oportuna para articular y formalizarla, precisamente porque - ya lo veremos - Deleuze basa su teoría del afecto en la de Spinoza.

Según la ley "espinosista", el afecto no puede afectar la naturaleza de lo que afecta. Es decir, para Spinoza, la relación de movimientos y reposos que me constituyen. Si un afecto afectara mi naturaleza misma, mi constitución psíquica o corpórea, ya no sería el mismo individuo que antes del afecto; el afecto no me habría afectado porque yo ya no sería yo. Puede haber cambios locales, en realidad tiene que haber cambios locales, pero un afecto que afectara mi naturaleza, que cambiara la relación entre mis movimientos y mis reposos, ya no sería afecto. Sería la muerte misma. ${ }^{13}$ Por eso, al describir la afectabilidad superior de los cuerpos de alta composición en el Lema 7 de la segunda parte de la Ética, Spinoza establece en dos ocasiones que no hay cambio alguno en la naturaleza del individuo afectado. El hecho que Spinoza observe la ley en frases subordinadas, no indica que la ley sea menos importante, sino que revela que es tan básica que no hace falta explicarla.

Por lo dicho, vemos, pues, cómo un individuo compuesto puede ser afectado de muchas maneras, conservando, no obstante, su naturaleza. [. . .] En efecto, supuesto que cada una de sus partes está compuesta de varios cuerpos, cada parte podrá [. . .], sin cambio alguno de su naturaleza, moverse más lenta o más rápidamente, $\mathrm{y}$, por consiguiente, comunicar sus movimientos a las otras más aprisa o más despacio (énfasis mío).

\footnotetext{
${ }^{12}$ Lo cual no impide que Deleuze y Guattari sigan siendo "humanistas" en otros respectos. El hecho de que el afecto se defina como el devenir no humano del ser humano ya lo señala en la medida en que el afecto aún se determina en términos humanos, aunque se trata de desplazar lo humano. Tal vez por la misma razón Deleuze y Guattari son más humanistas aún: el ser humano sigue rigiendo aún ahí donde se habrá encontrado derrocado.

13 "Aquí, de todas formas, debo observar que entiendo que la muerte del cuerpo sobreviene cuando sus partes quedan dispuestas de tal manera que alteran la relación de reposo y movimiento que hay entre ellas"' (Spinoza, Ética, Parte IV, Proposición 39, Escolio).
} 
Este individuo complejo, este individuo sumamente afectable y afectante, este individuo cuya mente supera las demás, este individuo es humano. Puede disfrutar de este privilegio afectivo precisamente porque la naturaleza humana no es afectada por los afectos.

Según la ley "deleuziana", en cambio, el afecto debe afectar la naturaleza misma de lo que afecta. Si no, si el afecto no afecta al afectado de alguna manera profunda y primordial, si el afectado sigue igual antes y después del afecto, "sin cambio alguno de su naturaleza" como dice Spinoza, entonces, a fin de cuentas, el afecto no habrá afectado nada. Ni siquiera habrá sido un afecto. El único afecto que no se puede descartar como secundario, accidental, insignificante, el único afecto irreductible, el único afecto que merece el nombre de "afecto", sería el que afecta esencialmente. El afecto que no afecta mi esencia no merece el nombre de "afecto"; en verdad, en la medida en que no es esencial, no merece ningún nombre. Sería un "afecto" sólo entre comillas, de modo metafórico o analógico, porque no es el afecto verdadero que afecta de verdad. Si opto por llamar a esta ley "deleuziana", por lo menos provisionalmente, es porque Deleuze parece observarla cuando define el afecto como "el devenir no humano del ser humano".

Las dos leyes antinómicas, entonces, son: por un lado, la ley "espinosista" que dicta que el afecto no puede afectar al afectado esencialmente; por otro lado, la ley deleuziana que dicta que el afecto debe afectar la esencia misma del afectado. Cada ley es tan imprescindible como irreductible. El afecto no puede afectar y no puede no afectar esencialmente. Es imposible observar una ley sin romper la otra. El afecto espinosista todavía no es afecto desde la perspectiva deleuziana, tal como el afecto deleuziano ya no es afecto desde la perspectiva espinosista. En ambos casos el único afecto no es afecto. Atrapada entre las dos leyes, el afecto debe ser más y menos que un afecto para ser un afecto. El afecto - la doble ley que esconde bajo la superficie calma de esa palabra tan de moda hoy en día- hace que toda experiencia sea criminal. Criminal por un crimen a priori, respecto del cual son cómplices Deleuze y Guattari, incluso si buscan una inocencia absoluta precisamente en nombre de Spinoza. ${ }^{14}$ De ahora en adelante, de todos modos, deberíamos confesar que no estamos tan seguros de qué hablamos cuando hablamos del "afecto".

\section{In-materialismo del afecto}

Ahora bien, fue a propósito que llamé a la segunda ley "deleuziana" sólo de manera provisional. No para eventualmente corregir o abolir la ley, sino en vistas a

${ }^{14}$ Cf. Deleuze y Guattari 1991 74/1993 74. 
que el afecto según Deleuze y Guattari - el devenir no humano del ser humanoes más sutil que lo que indica mi formulación algo bruta de la ley "deleuziana". Pareciera que Deleuze y Guattari toman en cuenta la otra ley, la que he llamado "espinosista". En el contexto de una discusión de la diferencia entre el devenir en la filosofía como disciplina creadora de conceptos y el devenir en el arte como disciplina creadora de afectos, Deleuze y Guattari escriben en ¿Qué es la filosofía?: "El devenir sensible es el acto a través del cual algo o alguien incesantemente se vuelve otro" -y agregan entre paréntesis - "(sin dejar de ser lo que es)" (1991 178/1993 179). Al devenir, me vuelvo otro (sin dejar de ser lo que soy). El paréntesis no debería engañar. Sin duda es una tentativa de callar, de suspender, de contener la ley espinosista. Pero la idea de que devengo sin dejar de ser lo que soy, se encuentra reafirmada cada vez que Deleuze y Guattari precisan que el devenir no es la transformación. Tampoco es la imitación, la simpatía, la identificación ni la semejanza ${ }^{15}$, pero sobre todo no es la transformación del ser humano en algo no humano. En Moby Dick, un ejemplo recurrente de Deleuze y Guattari, el capitán Acab deviene ballena sin que se transforme en una ballena. ¿Cómo es posible devenir sin llegar a ser, sin transformarse, sin morir? ¿Cómo hablar del devenir otro si lo que deviene sigue siendo lo que es? ¿Cómo puede el afecto hacerme devenir y dejarme ser? Si la metafísica desde Platón se despliega como el espacio infranqueable entre $e l$ ser y el devenir, pareciera que el afecto constituye una pregunta que ya no puede plantearse enteramente dentro de la metafísica. Esta es, tal vez, la dificultad más importante de y para la teoría deleuziana del afecto.

Todo gira en torno a la distinción fundamental entre el afecto y la afección: "los afectos ya no son sentimientos o afecciones, desbordan la fuerza de aquellos que pasan por ellos" (Deleuze y Guattari 1991 163-64/1993 165). Aunque Deleuze y Guattari no se refieren a Spinoza en su discusión del afecto en el séptimo capítulo de ¿Qué es la filosofía?, la distinción que hacen entre el afecto y la afección proviene de la tercera parte de la Ética, "Del origen y naturaleza de los afectos". En la tercera definición, Spinoza precisa: "Por afectos [Affectum] entiendo las afecciones [affectiones] del cuerpo, por las cuales aumenta o disminuye, es favorecida o perjudicada, la potencia de obrar de ese mismo cuerpo, y entiendo, al mismo tiempo, las ideas de esas afecciones". Cuando un cuerpo externo repercute en el mío, se trata de una afección, la cual aumenta o disminuye la potencia de obrar de mi cuerpo. La idea de una afección que aumenta mi potencia de obrar, me pone alegre. La que disminuye mi potencia de obrar, me pone triste. El deseo, a su vez, me lleva a perseguir todo lo que me ponga alegre, y evitar o destruir todo lo que me ponga triste. La alegría, la tristeza y el deseo constituyen los "afectos primarios" de

${ }^{15}$ Cf. Deleuze y Guattari 1991 173-74/1993 174-75. 
la Ética (Parte III, Proposición 11, Escolio). Este mecanismo del afecto, en breve, opera la eticidad misma de la Ética.

En Spinoza: filosofía práctica Deleuze se fija sobre todo en el aumento o disminución de potencia para marcar la diferencia entre el afecto y la afección. Pero en realidad no es la potencia en sí lo que le interesa a Deleuze. Más bien, por lo menos en cuanto a su propia teoría del afecto, le interesa la idea de un paso entre dos estados. "La affectio [afección] remite a un estado del cuerpo afectado e implica la presencia del cuerpo afectante, mientras que el affectus [afecto] remite al paso de un estado a otro distinto, considerada la variación de los cuerpos afectantes" (Deleuze 1981 67/2001 62-63). Debido a una afección, estoy en un estado determinado, digamos el estado A. De repente algo más me afecta, me aumenta o me disminuye la potencia de obrar, y ahora estoy en el estado B. En sentido estricto, según la lectura deleuziana, el afecto no es ni el estado $\mathrm{A}$ ni el estado $\mathrm{B}$, sino el paso de $A$ hacia $B$, el paso $A B$, el devenir $B$ de $A$ que no se reduce ni al estado A ni al estado B. El paso pasa entre los estados. Es esta idea de paso, de "entre", de devenir, la que Deleuze y Guattari adoptan para su propia teoría del afecto en ¿Qué es la filosofía?

Pero al apropiarse del afecto espinosista, del paso, del devenir, Deleuze efectúa una modificación importante que, por el momento por lo menos, no etiquetaré de ruptura.

Para Spinoza, el afecto se efectúa entre dos estados, entre dos afecciones, de un solo individuo. Aunque Spinoza aborda los afectos en la tercera parte de la Ética concentrándose en el individuo por excelencia, el ser humano, nada excluye que se trate de dos estados de un ser no humano, de otro animal, por ejemplo, pero el afecto espinosista siempre atañe a un solo individuo. Como el aumento o la disminución de la potencia de obrar de un cuerpo en particular, el afecto tiene un horizonte mono-corpóreo. Lo cual impone un límite esencial al devenir. A pesar de que no se sabe de antemano "lo que puede el cuerpo" (Ética, Parte III, Proposición 2, Escolio), una afirmación que la admiración de Deleuze ha hecho debidamente famosa, ${ }^{16}$ sí se sabe que el cuerpo no puede más que lo que le permiten sus "movimientos" y "reposos", su "ritmo", su "danza" en un sentido amplio. Por supuesto, como comentarista de Spinoza, Deleuze lo sabe bien. "Capacidad de afectos -escribe en Spinoza: filosofía práctica- con un umbral máximo y un umbral mínimo es una noción corriente en Spinoza" (1981 163/2001 151). Un afecto más allá del "umbral máximo" del cuerpo descompondría la relación entre movimientos y reposos que lo constituye, disolvería el cuerpo, pondría fin a su

\footnotetext{
${ }^{16} \mathrm{Cf}$, en particular, Spinoza y el problema de la expresión, capítulo 14, "¿Qué es lo que puede un cuerpo?" (Deleuze 1968 197-213/1996 208-225).
} 
danza. Lo que Deleuze llama "umbral máximo" es otra manera de describir la ley espinosista: lo que me afecta no me puede afectar de manera esencial.

Pareciera obvio que todo afecto le acontece a un solo cuerpo. Si eres la ocasión de mi alegría, no experimentas mi alegría, aunque tú también estés alegre. Pero es justamente este límite mono-corpóreo el que Deleuze pretende sobrepasar con su apropiación del afecto espinosista. Para Deleuze, el afecto es un paso entre dos estados, entre dos afecciones, tal como lo es para Spinoza. Pero Deleuze abre el afecto de modo que ya no es el paso entre dos estados del mismo individuo, sino entre dos individuos heterogéneos. Entre el ser humano y lo no humano, por ejemplo. "El afecto no es el paso de un estado vivido a otro -dicen Deleuze y Guattari-, sino el devenir no humano del hombre" (1991 173/1993 174). Aquí, en este momento preciso, al sacar el afecto de su límite mono-corpóreo, Deleuze y Guattari también lo sacan del horizonte espinosista. El afecto ya no es el paso de un estado vivido a otro, lo cual constituye la definición exacta del afecto espinosista según el propio Deleuze, sino el devenir no humano del ser humano, el devenir planta, por ejemplo, o el devenir animal. El paso entre dos estados para Spinoza, en breve, es el paso entre dos heterogeneidades para Deleuze y Guattari. De ahí que el afecto deleuziano se basa en el mismísimo afecto espinosista al que pareciera oponerse de manera antinómica.

En efecto, podemos apreciar de qué manera yo puedo volverme otro sin dejar de ser lo que soy, es decir, de qué manera el devenir no es una mera transformación. El afecto es lo que pasa entre dos individuos, dos cuerpos, dos heterogeneidades y por lo tanto no se reduce a ninguno de los dos. Se trata de una especie de exteriorización del afecto, la creación de una "zona de indeterminación" entre el afectador y el afectado en que, sin simplemente fusionarse, ya no se distinguen ${ }^{17}$. De las muchas consecuencias que siguen, en vistas del tiempo, me limito a señalar sólo dos, que me sirven como una doble conclusión. Mientras la primera consecuencia indica la radicalidad del afecto deleuziano, la segunda sugiere que Deleuze no se escapa de la tradición filosófica tan fácilmente que pareciera suponer.

Primera consecuencia. Si el afecto es un devenir que se efectúa entre dos individuos heterogéneos, en relación a los cuales permanece irreductible, entonces el afecto gana una autonomía insólita. Una autonomía que la tradición filosófica nunca ha podido ni querido otorgarle. A lo largo de la historia de la filosofía se supone que un ente o un ser determinado (un ser humano, por ejemplo) precede a las sensaciones que tiene. Pareciera lógico: tengo que ser, tengo que ser un ser, antes de que pueda ser afectado o afectar. Como resultado, la sensación es secundaria,

${ }_{17}$ Cf. Deleuze y Guattari 1991 174, 179/1993 175, 180. 
accidental, subordinada al ser que la "tiene". En realidad, la sensación es secundaria en doble sentido: secundaria al cuerpo que, a su vez, es secundario al alma o a la mente. Desde este punto de vista no hay gran diferencia entre el idealismo y el empirismo. Incluso cuando el empirismo es más empírico, por ejemplo, incluso cuando Hume determina la identidad personal como función de sentimiento, presupone un individuo ya constituido y capaz de sentir ${ }^{18}$. Pero Deleuze y Guattari rompen con la tradición al plantear que la sensación disfruta de una existencia autónoma que ya no depende de ningún ser que le preexiste. Para marcar esta autonomía acuñan una formulación contundente: un ser de sensación (1991 164/1993 165). No la sensación de un ser, sino un ser de la sensación. Así el afecto adquiere un estado ontológico: es un ser, es en un sentido fuerte ${ }^{19}$.

Segunda consecuencia. Sin embargo, sin quitarles a Deleuze y Guattari nada de su originalidad, nada de su fuerza, hay que destacar que ellos y sus acólitos logran autonomizar el afecto sólo a precio de la desincorporación, la desmaterialización, la idealización total del afecto. Por lo menos por derecho. El devenir afectivo que se efectúa entre dos cuerpos no puede se reduce a ninguno de los dos cuerpos. El devenir ballena de Acab no se encuentra ni en el cuerpo de Acab, ni en el cuerpo de la ballena, sino entre ellos. En realidad, habría que distinguir por lo menos tres cuerpos: los dos cuerpos que entran en un devenir, junto con el tercer cuerpo de la obra de arte que capta, que encarna, que crea el devenir. Acab, Moby Dick y las palabras, la sintaxis de Melville. Pero el afecto tampoco es el cuerpo del arte, no es la materia artística, dicen Deleuze y Guattari, "por lo menos por derecho" (1991 166/1993 167).

Sin duda, el afecto o la sensación en general siempre involucra idealidad de alguna forma. No hace falta saltar hasta el extremo platónico que busca reducir toda corporeidad en nombre de una Idea pura. Spinoza también ofrece un ejemplo: el afecto (affectus) es una afección (affectio) del cuerpo más la idea de esa afección (Ética, Parte III, Definición 3), de modo que la idea constituye la especificidad del afecto y su diferencia respecto a la afección. Lo cual sin embargo no permite, en cambio, que uno separe la idealidad del afecto de la afección sin correr el riesgo de secundarizar o accidentalizar el cuerpo. Así, de modo general, el gesto mismo con el

\footnotetext{
${ }^{18}$ De ahí el impasse que el propio Hume reconoce. $C f$. la famosa sección 6 de la cuarta parte del primer libro del Tratado de la naturaleza humana y el apéndice del mismo libro (especialmente 2000 400/1984 887-888). El primer libro de Deleuze, Empirismo y subjetividad (1953), consiste en una tentativa de superar este impasse.

19 Para uno de los estudios más influyentes de la llamada "teoría de afecto", que toma esta autonomía como punto de partida, ver "The Autonomy of Affect", capítulo primero de Parables for the Virtual de Brian Massumi.
} 
que Deleuze y Guattari romperían con la tradición - la autonomización del afecto - la repite.

Claro, en realidad, la reducción del cuerpo que se efectúa a lo largo de la tradición filosófica y la que se efectúa en la obra de Deleuze y Guattari no son iguales, de modo que Deleuze y Guattari no recaen simplemente en la tradición. Pero la diferencia entre la reducción tradicional y la deleuziana no disminuye la complicidad que Deleuze y Guattari tienen con la tradición en lo que dice relación a su tratamiento del cuerpo. Al contrario, la intensifica más aún.

La tradición, por su parte, reduce al cuerpo porque el cuerpo es reducible. El cuerpo, dice Platón incluso en su diálogo más sensual, "al que estamos encadenados" (Fedro 250c). Aunque el terreno cambia a lo largo de la historia de la filosofía, a fin de cuentas, el cuerpo no es nada, y, mal que bien, los filósofos no pierden nada cuando lo pierden. Todo lo contrario, suelen ganarlo todo. Platón constituye sólo un referente entre otros al respecto. Después de plantear la corporeidad como atributo divino, después de establecer la perspectiva sustancial desde la cual la mente es el cuerpo, después de plantear que la mente no es sino la idea del cuerpo ${ }^{20}$, la Ética alcanza la libertad humana en la Parte V, hacia la cual avanza desde el principio, sólo al abordar "la duración del alma [Mentis: de la mente], considerada ésta sin relación a la existencia del cuerpo [Corporis existentiam]" (Ética, Parte V, Proposición 20, Escolio, traducción modificada). Ninguna inmanencia separa a Spinoza de Platón en este punto ${ }^{21}$.

Deleuze y Guattari, en cambio, reducen al cuerpo a pesar de que el cuerpo es irreductible. Aunque el afecto que constituye la obra de arte es incorpóreo o inmaterial "por lo menos por derecho", Deleuze y Guattari plantean sin embargo que la obra de arte "incorpora" o "encarna" el acontecimiento virtual, "le confiere un cuerpo" (1991 178/1993 179). Por eso mismo, por lo menos por derecho, la reducción corpórea del afecto en ¿Qué es la filosofía? no tiene precedente. Algunos comentaristas reconocen e incluso discuten sobre el valor de la desincorporación deleuziana. Mientras unos la ven como el desamparo del cuerpo, otros la ven como la liberación del afecto $^{22}$. Las dos tendencias se encuentran justificadas, y, por lo tanto, ninguna es absolutamente correcta. Pues, paradójicamente, no a pesar o

\footnotetext{
${ }^{20}$ Cf. Spinoza, Ética, Parte I, Proposición 15, Escolio; Parte II, Proposición 7, Escolio; Parte II, Proposición 13.

${ }^{21}$ Sin cuestionarlo, el propio Deleuze reconoce el privilegio que goza el pensamiento en detrimento del cuerpo en el sistema espinosista. Cf. Deleuze 1981 92/2001 89 y el capitulo VII de Deleuze 1968/1996.

${ }^{22}$ Cf. "Affect as medium, or the digital-facial-image" de Marc Hansen, y "Response to Marc B. N. Hansen's 'Affect as medium, or the digital-facial-image", sendos artículos publicados en la Journal of Visual Culture.
} 
además de sino más bien precisamente debido a la radicalidad de su ruptura con la tradición, Deleuze y Guattari corren el riesgo de ser aún más tradicionales que la tradición misma. Su reducción reduce más y sacrifica más que todo lo que ha reducido la tradición en relación al cuerpo. De modo que uno podría plantear que sólo Deleuze y Guattari efectúan la reducción del cuerpo. Si al final el cuerpo no es nada para el filósofo de la tradición, pues el filósofo no reduce nada, y no le cuesta nada reducir al cuerpo. En cambio, cuando Deleuze y Guattari reducen al cuerpo, reducen al cuerpo en su irreductibilidad misma. En cierto modo contra la suposición de la fenomenología, de la cual tomo prestada esta idea de "reducción", se revela aquí que reducir lo reducible no es reducir nada, sólo lo irreductible es reductible, y sólo Deleuze y Guattari lo reducen.

Pero, por la misma razón, Deleuze y Guattari no reducen nunca al cuerpo de una vez por todas. La consecuencia, pues, es más brutal. Si, por derecho, el afecto es irreductible a toda materialidad, pero, de hecho, siempre hay encarnación, un plano material que sube e invade (envahir) el afecto ${ }^{23}$, pues el afecto es afecto sólo en la medida en que suprime el cuerpo una y otra vez. Por lo tanto, no basta con decir que Deleuze y Guattari no son tan materialistas como pretenden; ni siquiera basta con decir que son menos materialistas que el filósofo más idealista. En este sentido, la "conservación" por la cual Deleuze y Guattari alaban el arte - "es lo único en el mundo que se conserva" (1991 163/1993 164) - se conserva sólo al suprimir a todo cuerpo del mundo y por lo tanto a todo el mundo, y esta supresión se repite interminablemente. La conservación en el mundo es la destrucción repetida del mundo, y su nombre, en este caso, es arte.

\section{Bibliografía}

Deleuze, Gilles. Empirisme et subjetivité. Paris: PUF, 1953.

Deleuze, Gilles. Empirismo y subjetividad, trad. Hugo Acevedo. Barcelona: Editorial Gedisa, 2007.

Deleuze, Gilles. Spinoza et le problème de l'expression. París: Minuit, 1968.

Deleuze, Gilles. Spinoza y el problema de la expresión. Trad. Horst Vogel. Barcelona: Muchnik Editoriales, 1996.

Deleuze, Gilles Spinoza. Philosophie practique. París: Minuit, 1981.

Deleuze, Gilles. Spinoza: filosofía práctica, trad. Antonio Escohotado. Buenos Aires: Fábula, 2001.

Deleuze, Gilles. Critique et clinique. París: Minuit, 1993.

${ }^{23}$ Cf. Deleuze y Guattari 1991 166/1993 167. 
Deleuze, Gilles. Crítica y clínica, trad. Thomas Kauf. Barcelona: Editorial Anagrama, 1996.

Deleuze, Gilles y Félix Guattari. Qu'est-ce que la philosophie? París: Minuit, 1991.

Deleuze, Gilles y Félix Guattari. ¿Qué es la filosofía?, trad. Thomas Kauf. Barcelona: Anagrama, 1993.

Derrida, Jacques. Marges de la philosophie. París: Minuit, 1972.

Derrida, Jacques. Márgenes de la filosofía, trad. Carmen González Marín. Madrid: Cátedra, 1994.

Hansen, Mark. "Affect as medium, or the 'digital-facial-image'", Journal of visual culture 2/2 (2003): 205-228.

Hume, David. A Treatise of Human Nature. Ed. David Fate Norton and Mary J. Norton. Oxford: Oxford University Press, 2000.

Hume, David. Tratado de la naturaleza humana, trad. Félix Duque. Valenica: Ediciones Orbis, 1984.

Massumi, Brian. Parables for the Virtual: Movement, Affect, Sensation. Durham, NC: Duke University Press, 2002.

Platón. Fedro, trad. Armando Poratti. Madrid: Ediciones Akal, 2010.

Rushton, Richard. "Reponse to Mark B. N. Hansen's $<$ Affect as medium, or the 'digital-facial-image'>>", Journal of visual culture 3/3 (2004): 353-357.

Spinoza, Baruch. Ética demostrada según el orden geométrico, trad. Vidal Peña. Madrid: Alianza, 2017. 